\title{
Pembrolizumab in advanced osteosarcoma: results of a single-arm, open-label, phase 2 trial
}

\author{
Kjetil Boye ${ }^{1,2}(1) \cdot$ Alessandra Longhi $^{3} \cdot$ Tormod Guren $^{1} \cdot$ Susanne Lorenz ${ }^{4} \cdot$ Stine Næss $^{1} \cdot$ Michela Pierini $^{3}$. \\ Ingeborg Taksdal ${ }^{5}$. Ingvild Lobmaier ${ }^{6} \cdot$ Marilena Cesari $^{3} \cdot$ Anna Paioli $^{3}$ - Ayca M. Løndalen ${ }^{7}$. Elisabetta Setola ${ }^{3,8}$. \\ Ivar Hompland ${ }^{1} \cdot$ Leonardo A. Meza-Zepeda ${ }^{2,4} \cdot$ Kirsten Sundby Hall $^{1} \cdot$ Emanuela Palmerini $^{3}$
}

Received: 19 October 2020 / Accepted: 27 January 2021 / Published online: 12 February 2021

(c) The Author(s) 2021

\begin{abstract}
Aim To evaluate the activity and safety of the PD-1 antibody pembrolizumab in adult patients with advanced osteosarcoma. Material and methods The study was a single-arm, open-label, phase 2 trial in patients with unresectable, relapsed osteosarcoma. The primary endpoint was clinical benefit rate (CBR) at 18 weeks of treatment, defined as complete response, partial response, or stable disease using RECIST v1.1. The trial had a Simon's two-stage design, and $\geq 3$ of 12 patients with clinical benefit in stage 1 were required to proceed to stage 2 . The trial is registered with ClinicalTrials.gov, number NCT03013127. NanoString analysis was performed to explore tumor gene expression signatures and pathways.

Results Twelve patients were enrolled and received study treatment. No patients had clinical benefit at 18 weeks of treatment, and patient enrollment was stopped after completion of stage 1. Estimated median progression-free survival was 1.7 months (95\% CI 1.2-2.2). At time of data cut-off, 11 patients were deceased due to osteosarcoma. Median overall survival was 6.6 months (95\% CI 3.8-9.3). No treatment-related deaths or drug-related grade 3 or 4 adverse events were observed. PD-L1 expression was positive in one of 11 evaluable tumor samples, and the positive sample was from a patient with a mixed treatment response.

Conclusion In this phase 2 study in advanced osteosarcoma, pembrolizumab was well-tolerated but did not show clinically significant antitumor activity. Future trials with immunomodulatory agents in osteosarcoma should explore combination strategies in patients selected based on molecular profiles associated with response.
\end{abstract}

Keywords Osteosarcoma $\cdot$ Pembrolizumab $\cdot$ PD-1 inhibitor $\cdot$ PD-L1 expression $\cdot$ NanoString

\section{Introduction}

The prognosis for patients with recurrent osteosarcoma is poor [1-4]. Patients with a surgically resectable relapse may become long-term survivors, while recurrent, unresectable disease is almost always fatal [2]. Second-line systemic

Kjetil Boye

kjetil.boye@rr-research.no

1 Department of Oncology, Oslo University Hospital, The Norwegian Radium Hospital, PO Box 4953, NO-0424 Oslo, Norway

2 Department of Tumor Biology, The Norwegian Radium Hospital, Oslo University Hospital, Oslo, Norway

3 IRCCS Istituto Ortopedico Rizzoli, Bologna, Italy

4 Department of Core Facilities, The Norwegian Radium Hospital, Oslo University Hospital, Oslo, Norway treatment options include ifosfamide and etoposide with or without carboplatin, high-dose ifosfamide alone, or gemcitabine-based regimens [5, 6]. Tyrosine kinase inhibitors such as pazopanib, regorafenib, and cabozantinib also have significant anti-tumor activity [7-10] and represent alternatives to conventional chemotherapy. Still, systemic therapy

5 Department of Radiology, The Norwegian Radium Hospital, Oslo University Hospital, Oslo, Norway

6 Department of Pathology, The Norwegian Radium Hospital, Oslo University Hospital, Oslo, Norway

7 Department of Nuclear Medicine, The Norwegian Radium Hospital, Oslo University Hospital, Oslo, Norway

8 Department of Experimental, Diagnostic and Specialty Medicine, Alma Mater Studiorum, University of Bologna, Bologna, Italy 
in second and later lines seems to produce a limited prolongation in survival $[11,12]$, and new therapeutic approaches are needed.

Pembrolizumab is a highly selective humanized monoclonal antibody designed to directly block the interaction between the immune checkpoint programmed cell death protein 1 (PD-1) and its ligands PD-L1 and PD-L2, thereby enhancing anticancer T-cell activity. Pembrolizumab has shown durable antitumor activity in several solid tumor types and a favorable safety profile. The first study to systematically assess the activity of anti-PD-1-antibodies in sarcoma was the SARC028 trial [13]. In this phase 2 study, 22 osteosarcoma patients aged 12 years or older were included, of whom only one had an objective response. Further evidence on the lack of activity of immune checkpoint inhibition in osteosarcoma was recently provided from pediatric trials with pembrolizumab, nivolumab, and atezolizumab [14-16]. These three phase 1-2 trials had altogether 31 evaluable osteosarcoma patients, and none achieved a radiological response. In addition, a French study with metronomic cyclophosphamide and pembrolizumab reported partial response (PR) in one of 14 evaluable patients [17].

This study was designed to evaluate the antitumor activity and safety of pembrolizumab in adult patients with advanced osteosarcoma.

\section{Materials and methods}

\section{Study design and participants}

This study was a phase 2 , single arm, open-label, interventional trial of pembrolizumab in patients with advanced osteosarcoma entitled "PROMO: A phase II study of Pembrolizumab in patients with Relapsed Or Metastatic Osteosarcoma not eligible for curative surgery." Patients were eligible if they were aged 18 years or older, had histologically verified osteosarcoma of bone, had disease relapse or progression after at least one line of systemic treatment, were not eligible for curatively intended surgery, had an Eastern Cooperative Oncology Group performance status of 0 or 1 , had adequate bone marrow function (absolute neutrophil count $\geq 1500$ cells per $\mu \mathrm{L}$, platelets $\geq 100$ 000 per $\mu \mathrm{L}$, and hemoglobin $\geq 9 \mathrm{~g} / \mathrm{dL}$ ), renal function (creatinine $\leq 1.5 \mathrm{X}$ upper limit of normal (ULN) or glomerular filtration rate $\geq 60 \mathrm{~mL} / \mathrm{min}$ ), hepatic function (total bilirubin $\leq 1.5 \mathrm{X}$ ULN and aspartate transaminase and alanine transaminase $\leq 2.5 \times$ ULN and albumin $\geq 25 \mathrm{~g} / \mathrm{L}$ ), and coagulation function (international normalized ratio $\leq 1.5$ $\mathrm{X}$ ULN and activated partial thromboplastin time $\leq 1.5 \mathrm{X}$ ULN). Patients were ineligible if they had active central nervous system metastases, had an additional malignancy that was progressing or required active treatment, had autoimmune disease that required systemic treatment in the past 2 years, or had had prior treatment targeting PD-1 or PD-L1. The trial was registered with ClinicalTrials.gov, number NCT03013127, prior to inclusion of the first subject. The protocol was approved by the appropriate institutional review board and ethics committee and was conducted in accordance with the Declaration of Helsinki. All patients provided written informed consent before enrolment.

\section{Procedures}

Pembrolizumab $200 \mathrm{mg}$ was administered as a $30 \mathrm{~min}$ intravenous infusion on day 1 and repeated every 21 days. Adverse events were graded according to the Common Terminology Criteria for Adverse Events (CTCAE) version 4.0. Imaging was performed using computed tomography $(\mathrm{CT})$ scans every 6 weeks during treatment until week 18, and then every 9 weeks until progression of disease. Radiological response was assessed using the Response Evaluation Criteria in Solid Tumors (RECIST) v1.1. Optional 18F-fluorodeoxyglucose (FDG) positron emission tomography (PET)/CT imaging was scheduled at screening, week 6 and week 18. The tumor with the highest FDG uptake at baseline was used for measurement of maximum standardized uptake value (SUVmax). Metabolic response was evaluated with PET Response Criteria in Solid Tumors (PERCIST) v1.0 using SUVpeak based on body weight. Study biopsies were scheduled at screening and week 9. Patients were to complete EORTC QLQ-C30 questionnaires at screening and every 6 weeks during treatment.

\section{Outcomes}

The primary endpoint was clinical benefit rate (CBR), defined as complete response (CR), PR or stable disease (SD) 18 weeks after start of treatment. Secondary endpoints included progression-free survival (PFS), overall survival (OS), overall response rate (ORR), duration of response, response rates assessed by $18 \mathrm{~F}-\mathrm{FDG} \mathrm{PET} / \mathrm{CT}$, changes in health-related quality of life from baseline assessed using EORTC QLQ-C30, safety, and tolerability. PFS was measured from the date of first dose of pembrolizumab to the date of PD or death of any cause, whichever occurred first. Patients alive without PD at the time of their last study visit were censored at that date. OS was calculated from the date of first dose of pembrolizumab until date of death of any cause. Patients alive were censored at the time of their last survival follow-up visit. Data collection was locked as of April 15th 2020. 


\section{Statistical analysis}

The trial had a Simon's two-stage design. We assumed that a CBR after 18 weeks of $20 \%$ is a level of activity that is not of interest for further clinical development, whereas a CBR of $40 \%$ is of clinical interest. The type I error used was $10 \%$. The study had a power of $80 \%$ to reject the null hypothesis when the true CBR is $40 \%$. Planned accrual for the first stage was 12 patients. If there were $\leq 2$ patients with clinical benefit among these 12 patients, the study would be stopped. Otherwise, 13 additional patients were to be accrued for a total of 25 . The null hypothesis would be rejected if $\geq 8$ of the 25 fully evaluable patients derived clinical benefit. Survival was estimated using the Kaplan-Meier method.

\section{Immunohistochemistry}

Sections of $3 \mu \mathrm{m}$ were stained with hematoxylin and eosin and for PD-L1 expression. Immunohistochemical PD-L1 staining was performed using the Dako Omnis automated staining solution (Dako). Slides were pretreated using EnVision FLEX Target Retrieval Solution Low pH (Dako) and incubated with monoclonal anti-PD-L1 antibody (clone 22C3; Dako) for $40 \mathrm{~min}$ at $25{ }^{\circ} \mathrm{C}$. PD-L1 expression was evaluated by a sarcoma pathologist (I.L.), and categorized as $0,0-1,1-10,10-50$, and $>50 \%$ positive membranous staining of tumor cells.

\section{RNA isolation and gene expression analysis}

Total RNA from fresh frozen material was isolated using AllPrep DNA/RNA/miRNA Universal kit (Qiagen) following the manufacturer's protocol. The RNA was quantified with NanoDropTM One (Thermo Fisher Scientific), and RNA integrity was measured with the Bioanalyzer 2100 (Agilent). Gene expression analysis was performed on the NanoString nCounterTM Sprint Profiler (NanoString Technologies) with 50 ng total RNA starting material for each sample. For each sample, two replicates were hybridized over night $(16 \mathrm{~h})$ to the Pan Cancer IO 360 ${ }^{\mathrm{TM}}$ panel probe set. All twelve samples were further processed following the manufacturer's protocol. Analysis of the data was performed using the nSolverTM software version 4 (NanoString Technologies) and applying normalization based on housekeeping genes showing low variance across all samples.

\section{Results}

\section{Patients}

Between May 31st, 2017, and Sept 27th, 2018, 12 patients were enrolled, six at Oslo University Hospital in Oslo,
Norway and six at Rizzoli Orthopedic Institute in Bologna, Italy. Baseline patient characteristics are presented in Table 1. Median age was 43 years (range 19-55). Nine patients $(75 \%)$ had primary tumors in the lower extremity, two in the jaw, and one in the sacrum. All patients had distant metastasis at time of inclusion, and four (33\%) had metastatic disease at initial diagnosis. Median number of previous lines of chemotherapy was 3 (range 1-7).

\section{Treatment and radiological response}

The median number of cycles of pembrolizumab administered was 2 (range 1-6). Four patients received only one cycle, all due to clinical progression prior to the second cycle. Ten patients underwent at least one radiological evaluation, while two patients with clinical progression were not evaluated radiologically. PD was observed in 9 of 10 patients, of whom six had PD at the first evaluation after 6 weeks and three at the second evaluation after 12 weeks. One patient had SD at first evaluation and stopped study treatment after cycle 3 because steroid therapy was initiated due to dyspnea and hemoptysis caused by a progressive lung metastasis, and was not subsequently evaluated. Thus, 0 of 12 patients reached the primary endpoint of clinical benefit defined as SD, PR, or CR at 18 weeks of treatment. Best overall response is shown in Fig. 1. Due to rapid disease

Table 1 Baseline patient characteristics

\begin{tabular}{ll}
\hline & $\begin{array}{l}\text { Number } \\
\text { of patients } \\
(\%)^{*}\end{array}$ \\
\hline $\begin{array}{l}\text { Age, median (range) } \\
\text { Gender }\end{array}$ & $43(19-55)$ \\
Male & $8(67)$ \\
Female & $4(33)$ \\
Primary tumor localization & \\
Femur & $5(42)$ \\
Tibia & $4(33)$ \\
Other & $3(25)$ \\
Histological subtype & \\
Osteoblastic & $5(42)$ \\
Fibroblastic & $3(25)$ \\
Other & $4(33)$ \\
Performance status & \\
ECOG 0 & $9(75)$ \\
ECOG 1 & $3(25)$ \\
Previous lines of chemotherapy & \\
$\leq 2$ & $2(17)$ \\
3 & $6(50)$ \\
$\geq 4$ & $4(33)$ \\
\hline
\end{tabular}

*Unless otherwise specified. ECOG Eastern Cooperative Oncology Group. 


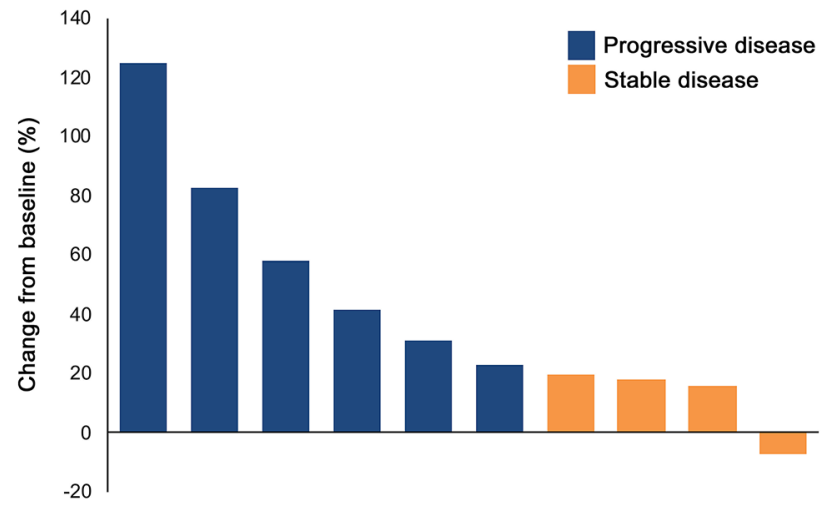

Fig. 1 Waterfall plot showing best RECIST response. Individual patients are represented by vertical bars and the change in tumor size according to RECIST v1.1 is depicted on the Y-axis. Two patients did not undergo radiological evaluation and are not included

progression, only four patients completed EORTC QLQ-C30 questionnaires during treatment, and quality of life results are thus not reported.

\section{Survival outcome}

Nine patients $(75 \%)$ had confirmed disease progression. Estimated median progression-free survival was 1.7 months (95\% CI 1.2-2.2). At time of data cut-off, 11 patients were deceased, all from osteosarcoma, and estimated median overall survival was 6.6 months (95\% CI 3.8-9.3).

\section{Adverse events}

Adverse events of grade 3 or higher occurred in 7 of 12 patients (58\%). Anemia grade 3 was reported in two patients, and increased alkaline phosphatase (grade 3), medullary compression (grade 3), pneumothorax (grade 3), and tumorrelated pain (grade 3 ) in one patient each. No treatmentrelated deaths or drug-related grade 3 or 4 adverse reactions were observed.

\section{F-FDG PET/CT}

Five patients underwent $18 \mathrm{~F}-\mathrm{FDG}$ PET/CT at baseline and after 6 weeks. The median SUVmax at baseline was 15.4 (range 4.1-20.5) and at first evaluation 11.4 (range 2.3-28.0). One patient had an increase in SUVmax from 20.5 to 28.0. SUVmax was reduced after 6 weeks in the other four patients, with an absolute reduction of 1.3-4.4 (12-44\%) compared to baseline values. Three patients had progressive metabolic disease (PMD) at first evaluation using PERCIST v1.0. One patient with stable metabolic disease (SMD) at the first evaluation had PMD at the second


Fig. 2 Photomicrographs of immunohistochemical staining with monoclonal anti-PD-L1 antibody (clone 22C3; Dako). a $>50 \%$ positive membranous staining of tumor cells. Scale bar $50 \mu \mathrm{m}$. b Negative staining. Scale bar $100 \mu \mathrm{m}$

PET/CT after 18 weeks, and one patient had SMD at both response evaluations.

\section{PD-L1 expression}

Pretreatment tumor samples from 11 patients were available for analysis of PD-L1 expression. From three patients tumor tissue was obtained by a study-specific biopsy before treatment and from 8 patients archival tumor material was used for the analyses. In one sample, there was a strong positive membranous expression of PD-L1 (> 50\% positive tumor cells), whereas the other ten samples were negative (Fig. 2). The PD-L1 positive specimen was a study biopsy of a soft tissue metastasis in the abdominal wall of a 41-year-old woman. She had PD and PMD after two treatment cycles, but with a mixed radiological and metabolic response. Uptake of 18F-FDG and tumor size were reduced in the lung and kidney metastases (Fig. 3, 


\section{A}
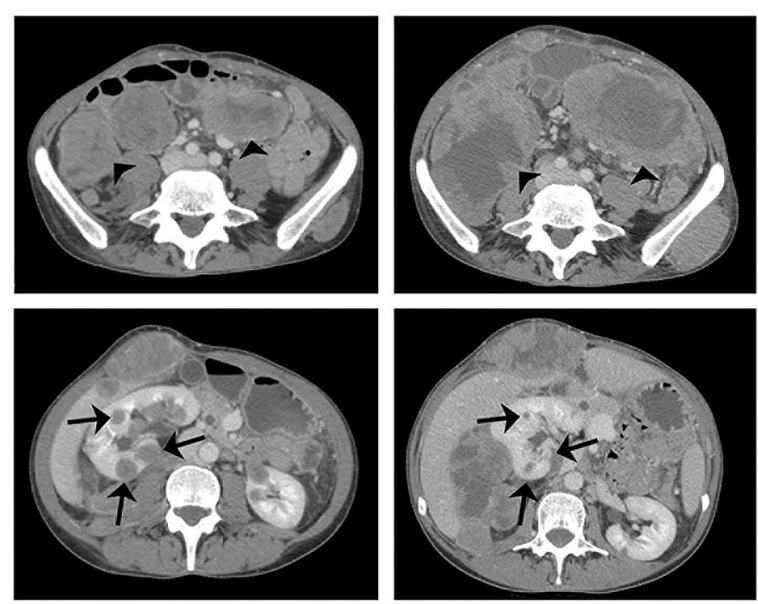

B
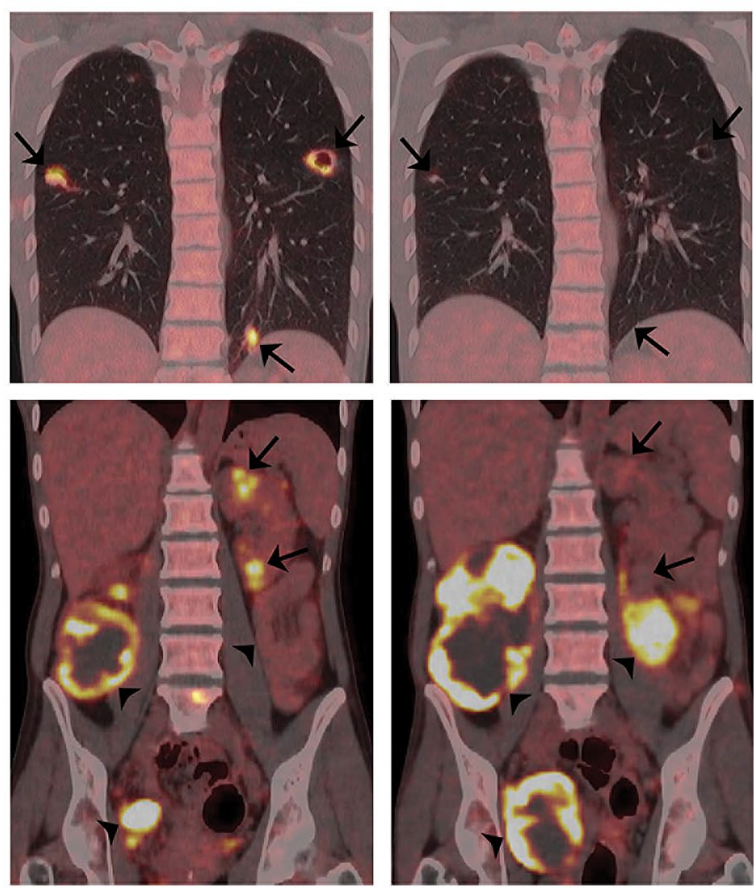

Fig. 3 Baseline images and response evaluation after treatment with pembrolizumab in study patient 12 (PROMO-12). a CT images and b 18F-FDG PET/CT images at baseline (left panel) and after two cycles of pembrolizumab (right panel). Arrows indicate reduced uptake of 18F-FDG and tumor size in lung and kidney metastases, and arrowheads indicate progressive abdominal and pelvic metastases

arrows), accompanied by an improved general condition and less tumor pain. There was, however, significant progression of abdominal and pelvic metastases (Fig. 3, arrowheads), and study treatment was discontinued.

\section{Gene expression analysis}

To explore gene expression signatures and pathways, six available fresh frozen tissue samples were analyzed on the NanoString nCounter platform using the PanCancer IO $360^{\mathrm{TM}}$ Panel. We compared the gene expression from the patient sample with mixed response (PROMO-12) with the other samples. The overall significantly upregulated genes $(p<0.01)$ were DUSP5, FOSL1, HMGA1, EROA1 and MET, and the significantly downregulated genes were $M A G E A 3 /$ $A 6$ and $H E Y 1$. Quantification of cell populations based on gene expression profiles revealed that PROMO-12 had a lower infiltration of mast cells; otherwise, no clear differences in immune cell infiltration between the samples was observed (Fig. 4a). To search for pathways associated with antitumor activity of PD-1 inhibition, we compared signature scores from PROMO-12 with the remaining samples. PROMO-12 had lower activity in four pathways: JAK-STAT signaling, NF-kB signaling, transforming growth factor- $\beta$ (TGF- $\beta$ ) signaling, and Wnt signaling (Fig. 4b). Three pathways showed higher activity: metabolic stress, epigenetic regulation, and Notch signaling (Fig. 4b). No clear differences were observed in the predicted activity of the other 18 pathways included in the analysis. Figure $4 \mathrm{c}$ shows the expression pattern of the most differentially expressed genes involved in the pathways with different activity levels in PROMO-12. Among these genes, we found the significantly upregulated genes DUSP5, FOSL1, HMGA1, EROA1, and $M E T$, and further increased expression of STAT1, CCND1, $C C N D 2$, and HIF1A. In total, we detected 17 genes with increased expression and 11 genes with decreased expression, contributing to the pathway scores of the pathways with higher or lower activity in PROMO-12.

\section{Discussion}

In this phase 2 study of adult patients with advanced osteosarcoma, pembrolizumab was well tolerated but did not show a clinically relevant antitumor activity. None of the 12 patients included in stage 1 of the trial achieved the primary endpoint of CR, PR, or SD at 18 weeks, and patient enrollment was thus stopped after completion of stage 1 .

Our findings align well with other studies of single-agent immune checkpoint inhibitors in advanced osteosarcoma [13-17] and confirm that osteosarcomas generally show primary resistance to treatment targeting PD-1/PD-L1, both in adult and pediatric patient cohorts. Nevertheless, there is antitumor activity of PD-1/PD-L1 inhibitors in some osteosarcoma patients. One of our patients had reduced FDG uptake and reduced size of all lung and kidney metastases, accompanied by reduced symptoms, and one patient in the SARC028 study had a long-lasting partial response [13]. 
A
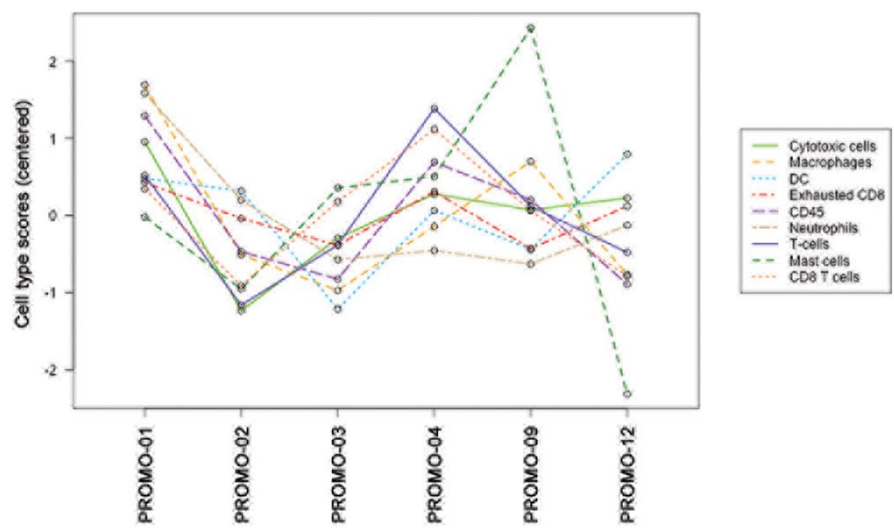

C

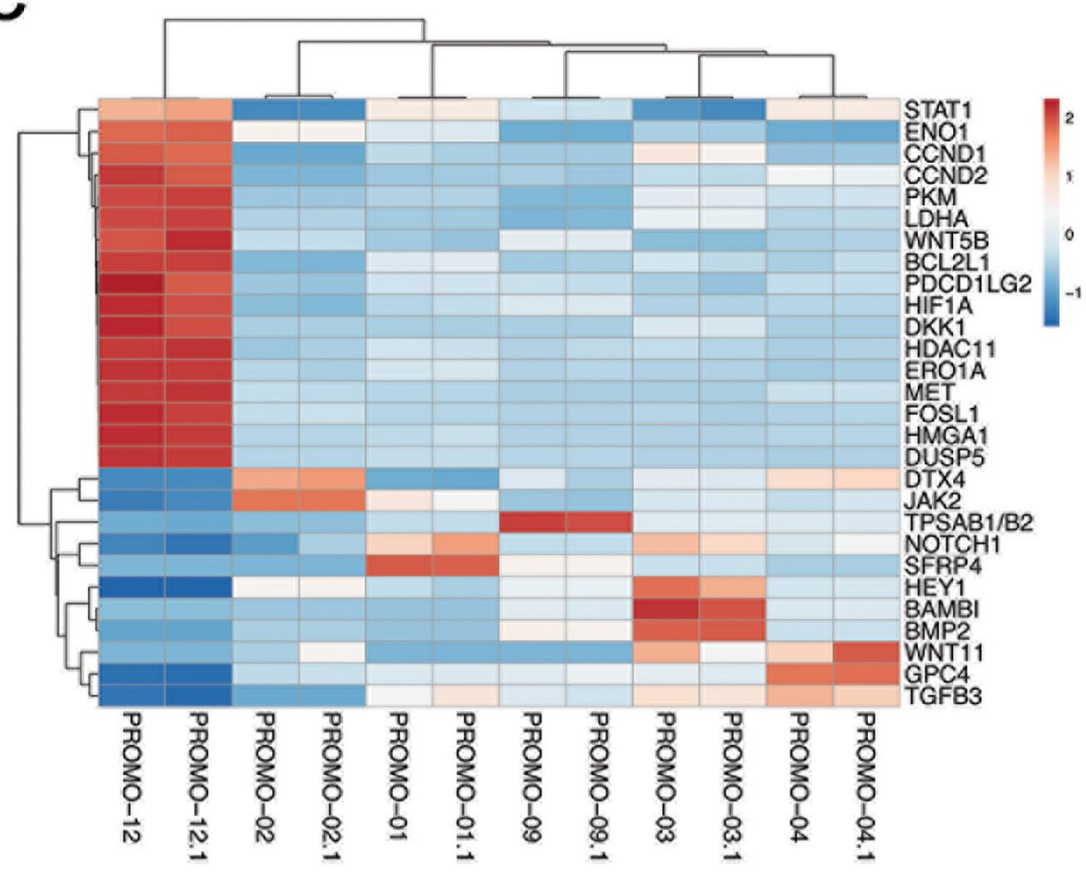

B

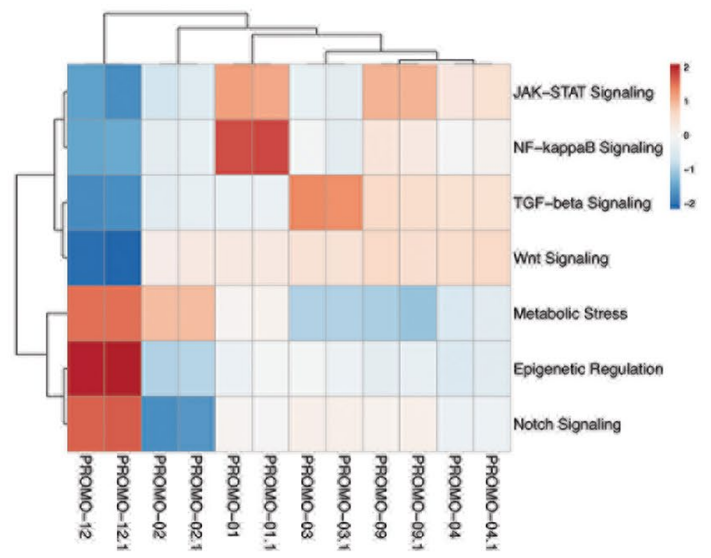

Fig. 4 Gene expression analysis of tumor samples using the NanoString platform. a Abundance of various cell type populations across different samples. The cell type score is calculated based on cell-type specific gene expression markers. b Pathways showing differential behavior in PROMO-12 compared to the remaining samples. Pathway scores are calculated based on gene expression data using nSolver V4 software and oriented such that increasing score corre-

Molecular profiling of samples from such patients may reveal pathways or markers predictive of response, or provide information about therapeutic resistance. In our study, a strong PD-L1 expression was observed in a pretreatment biopsy from the patient with a mixed response, whereas all other samples were PD-L1 negative, in agreement with previous studies [18]. In SARC028, only three of 70 evaluable samples were PD-L1 positive, all of which were sponds to increased expression. c Normalized gene expression values of top 28 differential expressed genes involved in these pathways. For both plots, rows are centered and unit variance scaling is applied to rows. Both rows and columns are clustered using correlation distance and average linkage. In $\mathbf{b}$ and $\mathbf{c}$, samples were analyzed in duplicate and both duplicates are shown

undifferentiated pleomorphic sarcomas [13]. Whether the responding osteosarcoma patient in that trial was among the evaluable samples was not specified. Although the overall predictive value of PD-L1 expression is uncertain in soft tissue sarcoma [19], the two patients with PD-L1 expression $>1 \%$ who were evaluable for response in the SARC028 trial had objective and durable responses. Furthermore, it is assumed that expression of PD-L1 is necessary, but perhaps 
not sufficient, for antitumor activity of immune checkpoint inhibitors targeting PD-1, and spatial and temporal variability in PD-L1 expression might explain response in tumors where no PD-L1 expression is detected in the examined tissue specimen. In PROMO-12, our patient with a mixed response, PD-L1 expression was strong both by immunohistochemistry and by mRNA expression analysis of a frozen specimen (data not shown), while all other investigated samples were negative.

TGF- $\beta$ and Wnt signaling activity were reduced in PROMO-12 compared to the other analyzed samples (Fig. 4b). Tumor-intrinsic signaling through these pathways is associated with an immunosuppressive microenvironment and low T-cell infiltration [20,21]. Thus, our findings might suggest that increased TGF- $\beta$ and Wnt signaling associated with an immunosuppressive tumor microenvironment contributes to the lack of response to pembrolizumab in osteosarcoma.

Identifying mechanisms of primary resistance and strategies to overcome resistance should be a focus of further studies in osteosarcoma. Several mechanisms might explain the observed primary resistance, including lack of recognition by the adaptive immune system, insensitivity to the antiproliferative and proapoptotic effects of T-cell effector molecules, and an immunosuppressive microenvironment $[22,23]$. A recent and comprehensive characterization of the immuno-genomic landscape of osteosarcoma suggests that the majority of osteosarcomas are "cold" tumors, with low levels of immune cell infiltration, low-to-moderate tumor mutation burden and lack of neoantigen expression [24]. Whole exome sequencing of four-matched tumor-normal pairs from our study was performed (data not shown), and confirmed a low tumor mutation burden with 6.6, 4.9, 5.3, and 2.6 single nucleotide variations ( $\mathrm{SNVs}$ ) per Mb. Combination strategies aimed at triggering immune cell activation and an immune-mediated antitumor response might be effective, such as the oncolytic virus talimogene laherparepvec that has entered clinical testing in soft tissue sarcoma [25].

In addition to combination strategies, enriching for patients who are more likely to benefit from immune checkpoint inhibition is probably necessary. Recently, it was shown that gene expression profiles associated with a high density of B cells and the presence of tertiary lymphoid structures predicted response to pembrolizumab in soft tissue sarcoma [26]. It will be of major interest to investigate whether these signatures are present in osteosarcoma samples, and if so, whether gene expression profiling could be used in future studies to select patients with a higher probability of response.

In conclusion, pembrolizumab was well-tolerated but had limited antitumor activity in adult patients with advanced osteosarcoma. None of the 12 patients in stage 1 of this phase 2 trial had clinical benefit, and patient enrollment was thus stopped. Future studies with immune checkpoint inhibitors in osteosarcoma should explore combination strategies in patients selected based on molecular profiles associated with response.

Acknowledgements We would like to thank Prof. Gunnar Sæter for support and advice in planning and initiation of the study. The study has received financial support from the Radium Hospital Foundation and Oslo University Hospital Trust. It was also supported in part by a research grant from the lnvestigator lnitiated Studies Program of Merck Sharp and Dohme Corp. NanoString services were provided by the Helse Sør-Ø $\varnothing$ st Genomics Core Facility (oslo.genomics.no) at Oslo University Hospital. The opinions expressed in this paper are those of the authors and do not necessarily represent those of Merck Sharp and Dohme Corp.

Author contribution $\mathrm{KB}$, TG, and $\mathrm{KSH}$ designed the study. $\mathrm{KB}$, AL, TG, SN, MP, IT, IL, MC, AP, AML, ES, IH, KSH, and EP were involved in patient inclusion, treatment and evaluation, and acquisition, interpretation and analysis of data. SL and LMZ were responsible for the NanoString analysis and molecular data interpretation. The first draft of the manuscript was written by KB, and all authors critically revised the manuscript. All authors read and approved the final version of the manuscript.

Funding Open access funding provided by University of Oslo (incl Oslo University Hospital).. The study has received financial support from the The Radium Hospital Foundation and Oslo University Hospital Trust. It was also supported in part by a research grant from the lnvestigator lnitiated Studies Program of Merck Sharp and Dohme Corp.

\section{Compliance with ethical standards}

Conflict of interest K. Boye has served on advisory board for Bayer and has received research support from Eli Lilly and Merck. E. Palmerini has served on an advisory board for Takeda, Amgen, Daiichi Sankyo, Eli Lilly, Eusa Pharma, and Deciphera, and has received other research support from Bristol Myers Squibb, Pfizer, PharmaMar, and Daiichi Sankyo.

Ethical approval The study was approved by the institutional review boards and ethics committees of the involved institutions. It was conducted in accordance with the Declaration of Helsinki and according to Good Clinical Practice. The trial was registered with ClinicalTrials. gov, number NCT03013127, and all patients provided written informed consent before enrolment.

Open Access This article is licensed under a Creative Commons Attribution 4.0 International License, which permits use, sharing, adaptation, distribution and reproduction in any medium or format, as long as you give appropriate credit to the original author(s) and the source, provide a link to the Creative Commons licence, and indicate if changes were made. The images or other third party material in this article are included in the article's Creative Commons licence, unless indicated otherwise in a credit line to the material. If material is not included in the article's Creative Commons licence and your intended use is not permitted by statutory regulation or exceeds the permitted use, you will need to obtain permission directly from the copyright holder. To view a copy of this licence, visit http://creativecommons.org/licenses/by/4.0/. 


\section{References}

1. Bacci G, Briccoli A, Longhi A, Ferrari S, Mercuri M, Fagioli F et al (2005) Treatment and outcome of recurrent osteosarcoma: experience at Rizzoli in 235 patients initially treated with neoadjuvant chemotherapy. Acta Oncol 44(7):748-755. https://doi. org/10.1080/02841860500327503

2. Bielack SS, Kempf-Bielack B, Branscheid D, Carrle D, Friedel G, Helmke K et al (2009) Second and subsequent recurrences of osteosarcoma: presentation, treatment, and outcomes of 249 consecutive cooperative osteosarcoma study group patients. J Clin Oncol 27(4):557-565. https://doi.org/10.1200/JCO.2008.16.2305

3. Saeter G, Hoie J, Stenwig AE, Johansson AK, Hannisdal E, Solheim OP (1995) Systemic relapse of patients with osteogenic sarcoma. prognostic factors for long term survival. Cancer 75(5):1084-1093

4. Tirtei E, Asaftei SD, Manicone R, Cesari M, Paioli A, Rocca M et al (2018) Survival after second and subsequent recurrences in osteosarcoma: a retrospective multicenter analysis. Tumori 104(3):202-206. https://doi.org/10.1177/0300891617753257

5. Casali PG, Bielack S, Abecassis N, Aro HT, Bauer S, Biagini R et al (2018) Bone sarcomas ESMO-PaedCan-EURACAN Clinical Practice Guidelines for diagnosis treatment and follow up. Ann Oncol 29: 79-95

6. Palmerini E, Jones RL, Marchesi E, Paioli A, Cesari M, Longhi A et al (2016) Gemcitabine and docetaxel in relapsed and unresectable high-grade osteosarcoma and spindle cell sarcoma of bone. BMC Cancer. https://doi.org/10.1186/s12885-016-2312-3

7. Davis LE, Bolejack V, Ryan CW, Ganjoo KN, Loggers ET, Chawla S et al (2019) Randomized double-blind phase II study of regorafenib in patients with metastatic osteosarcoma. J Clin Oncol 37(16):1424-1431. https://doi.org/10.1200/JCO.18.02374

8. Duffaud F, Mir O, Boudou-Rouquette P, Piperno-Neumann S, Penel N, Bompas E et al (2019) Efficacy and safety of regorafenib in adult patients with metastatic osteosarcoma: a non-comparative, randomised, double-blind, placebo-controlled, phase 2 study. Lancet Oncol 20(1):120-133. https://doi.org/10.1016/S1470 -2045(18)30742-3

9. Italiano A, Mir O, Mathoulin-Pelissier S, Penel N, Piperno-Neumann S, Bompas E et al (2020) Cabozantinib in patients with advanced Ewing sarcoma or osteosarcoma (CABONE): a multicentre, single-arm, phase 2 trial. Lancet Oncol 21(3):446-455. https://doi.org/10.1016/S1470-2045(19)30825-3

10. Longhi A, Paioli A, Palmerini E, Cesari M, Abate ME, Setola E et al (2019) Pazopanib in relapsed osteosarcoma patients: report on 15 cases. Acta Oncol 58(1):124-128. https://doi. org/10.1080/0284186X.2018.1503714

11. Ferrari S, Briccoli A, Mercuri M, Bertoni F, Picci P, Tienghi A et al (2003) Postrelapse survival in osteosarcoma of the extremities: prognostic factors for long-term survival. J Clin Oncol 21(4):710-715. https://doi.org/10.1200/JCO.2003.03.141

12. Kempf-Bielack B, Bielack SS, Jurgens H, Branscheid D, Berdel WE, Exner GU et al (2005) Osteosarcoma relapse after combined modality therapy: an analysis of unselected patients in the cooperative osteosarcoma study group (COSS). J Clin Oncol 23(3):559-568. https://doi.org/10.1200/JCO.2005.04.063

13. Tawbi HA, Burgess M, Bolejack V, Van Tine BA, Schuetze SM, $\mathrm{Hu} \mathrm{J}$ et al (2017) Pembrolizumab in advanced soft-tissue sarcoma and bone sarcoma (SARC028): a multicentre, two-cohort, singlearm, open-label, phase 2 trial. Lancet Oncol 18(11):1493-1501. https://doi.org/10.1016/S1470-2045(17)30624-1

14. Davis KL, Fox E, Merchant MS, Reid JM, Kudgus RA, Liu $\mathrm{X}$ et al (2020) Nivolumab in children and young adults with relapsed or refractory solid tumours or lymphoma (ADVL1412): a multicentre, open-label, single-arm, phase 1-2 trial. Lancet Oncol 21(4):541-550. https://doi.org/10.1016/S1470-2045(20)30023-1

15. Geoerger B, Kang HJ, Yalon-Oren M, Marshall LV, Vezina C, Pappo A et al (2020) Pembrolizumab in paediatric patients with advanced melanoma or a PD-L1-positive, advanced, relapsed, or refractory solid tumour or lymphoma (KEYNOTE-051): interim analysis of an open-label, single-arm, phase 1-2 trial. Lancet Oncol 21(1):121-133. https://doi.org/10.1016/S1470 -2045(19)30671-0

16. Geoerger B, Zwaan CM, Marshall LV, Michon J, Bourdeaut F, Casanova $\mathrm{M}$ et al (2020) Atezolizumab for children and young adults with previously treated solid tumours, non-Hodgkin lymphoma, and Hodgkin lymphoma (iMATRIX): a multicentre phase 1-2 study. Lancet Oncol 21(1):134-144. https://doi.org/10.1016/ S1470-2045(19)30693-X

17. Le Cesne A, Marec-Berard P, Blay JY, Gaspar N, Bertucci F, Penel N et al (2019) Programmed cell death 1 (PD-1) targeting in patients with advanced osteosarcomas: results from the PEMBROSARC study. Eur J Cancer 119:151-157. https://doi. org/10.1016/j.ejca.2019.07.018

18. Palmerini E, Agostinelli C, Picci P, Pileri S, Marafioti T, Lollini PL et al (2017) Tumoral immune-infiltrate (IF), PD-L1 expression and role of CD8/TIA-1 lymphocytes in localized osteosarcoma patients treated within protocol ISG-OS1. Oncotarget 8(67):111836-111846. https://doi.org/10.18632/oncotarget.22912

19. Martin-Broto J, Moura DS, Van Tine BA (2020) Facts and hopes in immunotherapy of soft tissue sarcomas. Clin Cancer Res. https ://doi.org/10.1158/1078-0432.CCR-19-3335

20. Li X, Xiang Y, Li F, Yin C, Li B, Ke X (2019) WNT/betacatenin signaling pathway regulating $\mathrm{T}$ cell-inflammation in the tumor microenvironment. Front Immunol 10:2293. https://doi. org/10.3389/fimmu.2019.02293

21. Lind H, Gameiro SR, Jochems C, Donahue RN, Strauss J, Gulley JM, et al. (2020) Dual targeting of TGF-beta and PD-L1 via a bifunctional anti-PD-L1/TGF-betaRII agent: status of preclinical and clinical advances. J Immunother Cancer 8:1. doi: https://doi. org/10.1136/jitc-2019-000433

22. Ochoa de Olza M, Navarro Rodrigo B, Zimmermann S, Coukos G (2020) Turning up the heat on non-immunoreactive tumours: opportunities for clinical development. Lancet Oncol 21(9):e419e430. https://doi.org/10.1016/S1470-2045(20)30234-5

23. Syn NL, Teng MWL, Mok TSK, Soo RA (2017) De-novo and acquired resistance to immune checkpoint targeting. Lancet Oncol 18(12):e731-e741. https://doi.org/10.1016/S1470-2045(17)30607 $-1$

24. Wu CC, Beird HC, Andrew Livingston J, Advani S, Mitra A, Cao $S$ et al (2020) Immuno-genomic landscape of osteosarcoma. Nat Commun 11(1):1008. https://doi.org/10.1038/s41467-020-14646 $-\mathrm{W}$

25. Kelly CM, Antonescu CR, Bowler T, Munhoz R, Chi P, Dickson MA et al (2020) Objective response rate among patients with locally advanced or metastatic sarcoma treated with talimogene laherparepvec in combination with pembrolizumab: a phase 2 clinical trial. JAMA Oncol 6(3):402-408. https://doi.org/10.1001/ jamaoncol.2019.6152

26. Petitprez F, de Reynies A, Keung EZ, Chen TW, Sun CM, Calderaro J et al (2020) B cells are associated with survival and immunotherapy response in sarcoma. Nature 577(7791):556-560. https ://doi.org/10.1038/s41586-019-1906-8

Publisher's Note Springer Nature remains neutral with regard to jurisdictional claims in published maps and institutional affiliations. 